\title{
ON SIDE CONSTRAINED MODELS OF TRAFFIC EQUILIBRIA
}

\author{
Torbjörn Larsson ${ }^{1}$ and Michael Patriksson ${ }^{2}$ \\ Division of Optimization \\ Department of Mathematics \\ Linköping Institute of Technology \\ S-581 83 Linköping, Sweden \\ 1 e-mail: tl@math.liu.se \\ 2 e-mail: mipat@math.liu.se
}

\begin{abstract}
The basic model of traffic assignment does not capture complex traffic flow relationships such as interactions among vehicles on different road links, joint capacities on two-way streets, turning priorities in junctions, etc. Traditionally, such relationships are introduced implicitly through generalizations of the separable travel cost function of the basic model, and the refined models are usually stated as variational inequalities. However, this approach has found very little practical use, seemingly mostly because the generalized travel cost functions are not easily chosen and calibrated.

An alternative strategy for refining the basic model is to capture supplementary traffic flow relationships explicitly through the introduction of side constraints. This explicit approach has the advantage of always leading to optimization formulations, and the immediate physical interpretations of the side constraints introduced may make it easy to construct, calibrate and apply the resulting models compared to models with non-separable cost functions.

We consider a traffic equilibrium model with general side constraints and show that its optimality conditions correspond to a generalization of Wardrop's user equilibrium principle. Further, we derive a close relationship between models of traffic equilibria involving side constraints and generalized travel cost functions, respectively. These results motivate a further study into the art of modelling traffic assignment problems through the use of explicit side constraints.
\end{abstract}

Key Words: Traffic assignment, user equilibrium, side constraints, generalized Wardrop conditions, variational inequalities. 


\section{INTRODUCTION AND MOTIVATION}

Consider a transportation network $\mathcal{G}=(\mathcal{N}, \mathcal{A})$ of nodes and directed links. Between certain pairs of origins and destinations, $(p, q) \in \mathcal{C}$, fixed positive travel demands $d_{p q}$ are given, and each link $a \in \mathcal{A}$ is associated with a positive and strictly increasing travel cost function $t_{a}: \Re_{+}^{|\mathcal{A}|} \mapsto \Re_{++}$. The user equilibrium principle of Wardrop (1952) states that for each origin-destination $(\mathrm{O}-\mathrm{D})$ pair $(p, q)$, the routes utilized have equal and minimal travel costs, that is, at an equilibrium flow, the conditions

$$
\begin{aligned}
& h_{p q r}>0 \Longrightarrow c_{p q r}=\pi_{p q}, \quad \forall r \in \mathcal{R}_{p q}, \\
& h_{p q r}=0 \Longrightarrow c_{p q r} \geq \pi_{p q}, \quad \forall r \in \mathcal{R}_{p q} \text {, }
\end{aligned}
$$

where $h_{p q r}$ is an equilibrium flow on route $r \in \mathcal{R}_{p q}, c_{p q r}=c_{p q r}(h)$, with $h=\left(h_{p q r}\right)$, is the travel cost on the route, and $\pi_{p q}$ is the equilibrium travel cost of the least-cost routes in O-D pair $(p, q)$, are satisfied.

For separable travel cost functions, i.e., where the travel cost on each link is independent of the flow on other links, a solution to the Wardrop Conditions (1.1)-(1.2) can be found by solving the convex network optimization problem (e.g., Beckmann et al., 1956, and Dafermos, 1972)

$[\mathrm{TAP}]$

$$
\min T(f) \stackrel{\text { def }}{=} \sum_{a \in \mathcal{A}} \int_{0}^{f_{a}} t_{a}(s) d s
$$

subject to

$$
\begin{aligned}
\sum_{r \in \mathcal{R}_{p q}} h_{p q r} & =d_{p q}, \quad \forall(p, q) \in \mathcal{C} \\
h_{p q r} & \geq 0, \quad \forall r \in \mathcal{R}_{p q}, \quad \forall(p, q) \in \mathcal{C}, \\
\sum_{(p, q) \in \mathcal{C}} \sum_{r \in \mathcal{R}_{p q}} \delta_{p q r a} h_{p q r} & =f_{a}, \quad \forall a \in \mathcal{A},
\end{aligned}
$$

where

$$
\delta_{p q r a} \stackrel{\text { def }}{=} \begin{cases}1, & \text { if route } r \in \mathcal{R}_{p q} \text { uses link } a, \quad \forall a \in \mathcal{A}, \forall r \in \mathcal{R}_{p q}, \forall(p, q) \in \mathcal{C}, \\ 0, & \text { otherwise },\end{cases}
$$

is the link-route incidence matrix, and $f_{a}$ denotes the total flow on link $a$.

The equilibrium model [TAP] is frequently applied in transportation analysis, and many algorithms have been developed for its efficient solution. (See, e.g., Patriksson, 1995, for a thorough review of algorithms for [TAP].) The inherent simplicity of the model, however, makes it inapplicable to more complex traffic problems (e.g., Sender and Netter, 1970). For instance, it does not capture the interactions between the flows on intersecting links, or between vehicles of different types. An illustrative example of a deficiency of the model and its possible consequences is provided by Hearn (1980), who comments on its property of allowing every road to carry arbitrarily large volumes of traffic. This deficiency of the model causes that

"the predicted flow on some links will be far lower or far greater than the traffic engineer knows they should be if all assumptions of the model are correct. In practice, the result is that the model predictions are ignored, or, more often, the user will perturb the components of the model (trip table, volume delay formulas, etc.) in an attempt to bring the model output more in line with the anticipated results." 
In order to avoid such heuristic tampering with components of the model available, traffic planners must be supplied with analysis tools whose underlying traffic models are sufficiently general, reliable and accurate.

We distinguish two approaches for improving the model's ability to accurately describe, reproduce, or predict a real-world traffic situation.

The traditional approach is to capture additional flow relationships through the introduction of non-separable, and typically also asymmetric, travel cost functions. The solution of (1.1)-(1.2) can then, however, not be reformulated into an optimization problem of the form [TAP], due to the non-integrability of the resulting travel cost function $c: \Re_{+}^{|\mathcal{A}|} \mapsto \Re_{++}^{|\mathcal{A}|}$. Instead, the Wardrop conditions are formulated as the variational inequality problem of finding an $f^{*} \in F$ such that

[VIP]

$$
c\left(f^{*}\right)^{\mathrm{T}}\left(f-f^{*}\right) \geq 0, \quad \forall f \in F,
$$

where $F=\left\{f \in \Re^{|\mathcal{A}|} \mid f\right.$ satisfies (1.4)-(1.6) $\}$. The resulting class of models has been extensively studied from a theoretical and algorithmical point of view (see, e.g., Nagurney, 1993, and Patriksson, 1995). Seemingly, the asymmetric models' popularity is a consequence of their mathematical elegance and nice interpretations rather than their applicability, since real-world applications seem to be lacking. A major reason for this is probably the practical difficulty of choosing and calibrating the asymmetric travel cost functions.

An alternative - but so far little studied - approach to improve the quality of the basic traffic equilibrium model is to introduce a set of side constraints to model additional restrictions on possible flow patterns. Such side constraints could be used to describe, for instance, the interactions among vehicles in a junction, joint capacities on two-way streets and links in intersections and roundabouts, requirements that observed flows on some links should be reproduced in the calculated solution, a traffic control policy, or dynamic aspects. We believe this approach to be appealing from a practical point of view, since it is certainly easier for the traffic engineer to identify a suitable set of side constraints - which may have immediate physical interpretations - than to estimate proper values of parameters in complex travel cost functions. (In the example provided by Hearn (1980), the proper improvement of the basic model is the introduction of link capacity constraints corresponding to the engineer's anticipation of reasonable levels of traffic flow.) The approach to improve a traffic equilibrium model by introducing general side constraints was first discussed by Larsson and Patriksson (1994).

Although this alternative approach is more straightforward than that based on asymmetric cost functions, it has been given comparatively very limited attention. We present a general side constrained assignment model and investigate its optimality conditions; these may be interpreted as a generalization of Wardrop's equilibrium Principle (1.1)-(1.2) in the sense that an equilibrium holds in terms of generalized travel costs. Moreover, we show that the side constrained assignment problem may be equivalently solved as a standard equilibrium model with a well-defined adjusted travel cost function. This result leads to an interesting relationship between side constrained and asymmetric models of traffic equilibria, which is one motivation for further studying side constrained models. 


\section{A SIDE CONSTRAINED ASSIGNMENT MODEL}

Let $g_{k}: \Re_{+}^{|\mathcal{A}|} \mapsto \Re, k \in \mathcal{K}$, be convex and continuously differentiable, and define the side constraints

$$
g_{k}(f) \leq 0, \quad \forall k \in \mathcal{K} .
$$

Here, the index set $\mathcal{K}$ may, for instance, consist of the index set of the links, nodes, routes, or O-D pairs, or any combination of subsets of them. (The constraints are, without any loss of generality, given as inequalities.)

Consider the general side constrained traffic equilibrium problem

[TAP-SC]

$$
\min T(f)=\sum_{a \in \mathcal{A}} \int_{0}^{f_{a}} t_{a}(s) d s
$$

subject to

$$
\begin{aligned}
\sum_{r \in \mathcal{R}_{p q}} h_{p q r} & =d_{p q}, \quad \forall(p, q) \in \mathcal{C}, \\
h_{p q r} & \geq 0, \quad \forall r \in \mathcal{R}_{p q}, \quad \forall(p, q) \in \mathcal{C}, \\
\sum_{(p, q) \in \mathcal{C}} \sum_{r \in \mathcal{R}_{p q}} \delta_{p q r a} h_{p q r} & =f_{a}, \quad \forall a \in \mathcal{A}, \\
g_{k}(f) & \leq 0, \quad \forall k \in \mathcal{K} .
\end{aligned}
$$

We assume that the feasible set of [TAP-SC] is non-empty; in cases where some functions $g_{k}$ are nonlinear, we also assume that a constraint qualification (e.g., Bazaraa et al., 1993, Ch. 5) holds. The convexity of [TAP-SC] then ensures the existence of an optimal solution, which is unique in the link flows and characterized by the first-order optimality conditions. We next show that the optimality conditions of [TAP-SC] give rise to a Wardrop equilibrium principle in terms of generalized route travel costs.

Theorem 1. (A generalization of the Wardrop principle) If $(h, f)$ solves the problem [TAP-SC] and $\pi \in \Re^{|\mathcal{C}|}$ and $\beta \in \Re^{|\mathcal{K}|}$ are vectors of optimal Lagrange multipliers for the Constraints (2.2) and (2.5), respectively, then

$$
\begin{aligned}
& h_{p q r}>0 \Longrightarrow \bar{c}_{p q r}=\pi_{p q}, \quad \forall r \in \mathcal{R}_{p q}, \\
& h_{p q r}=0 \Longrightarrow \bar{c}_{p q r} \geq \pi_{p q}, \quad \forall r \in \mathcal{R}_{p q}
\end{aligned}
$$

holds for all $O-D$ pairs $(p, q) \in \mathcal{C}$, where

$$
\bar{c}_{p q r} \stackrel{\text { def }}{=} c_{p q r}(h)+\sum_{a \in \mathcal{A}} \delta_{p q r a}\left(\sum_{k \in \mathcal{K}} \beta_{k} \frac{\partial g_{k}(f)}{\partial f_{a}}\right), \quad \forall r \in \mathcal{R}_{p q}, \quad \forall(p, q) \in \mathcal{C} .
$$

Proof. Stating the stationary point conditions for the Lagrangean function

$$
L(f, \beta) \stackrel{\text { def }}{=} T(f)+\sum_{k \in \mathcal{K}} \beta_{k} g_{k}(f)
$$


subject to $(2.2)-(2.4)$ we obtain, from the convexity of [TAP-SC], that $(h, f)$ is a solution if and only if

$$
\begin{array}{rlrl}
h_{p q r}\left(\bar{c}_{p q r}-\pi_{p q}\right) & =0, & \forall r \in \mathcal{R}_{p q}, \quad \forall(p, q) \in \mathcal{C}, \\
\bar{c}_{p q r}-\pi_{p q} \geq 0, & \forall r \in \mathcal{R}_{p q}, \quad \forall(p, q) \in \mathcal{C}, \\
\sum_{r \in \mathcal{R}_{p q}} h_{p q r} & =d_{p q}, & & \forall(p, q) \in \mathcal{C}, \\
h_{p q r} & \geq 0, & & \forall r \in \mathcal{R}_{p q}, \forall(p, q) \in \mathcal{C}, \\
\sum_{(p, q) \in \mathcal{C}} \sum_{r \in \mathcal{R}_{p q}} \delta_{p q r a} h_{p q r} & =f_{a}, & & \forall a \in \mathcal{A}, \\
\beta_{k} g_{k}(f) & =0, & & \forall k \in \mathcal{K}, \\
g_{k}(f) & \leq 0, & \forall k \in \mathcal{K}, \\
\beta_{k} & \geq 0, & \forall k \in \mathcal{K},
\end{array}
$$

where $\bar{c}_{p q r}$ is given by (2.8).

The Condition (2.11), together with (2.10) and (2.12), implies that the multiplier $\pi_{p q}$ is the minimum generalized travel cost $\bar{c}_{p q r}$ in O-D pair $(p, q)$, and (2.10) further states that these costs are equal for all routes utilized in the O-D pair. Hence, the Conditions (2.10)-(2.11) imply (2.6)-(2.7), and the theorem is proved.

Solutions to [TAP-SC] thus correspond to flows satisfying a generalization of the Wardrop equilibrium conditions, based on the generalized travel Costs (2.8).

One can, in general, not relate the actual travel costs of the unused routes to those of the used ones; for instance, the cheapest route in an O-D pair may be unused because its generalized cost is too high. Furthermore, the Wardrop principle for [TAP] is intimately associated with the Cartesian product structure of its feasible set, and one can for the side constrained model not state similar optimality conditions in terms of actual travel costs. However, under the (reasonable) additional assumption that each constraint function $g_{k}$ is nondecreasing in each link flow variable $f_{a}$, a Wardrop-type principle in terms of actual travel costs may be established. (This result generalizes that of Larsson and Patriksson, 1994, for the case of link flow capacity side constraints.)

Theorem 2. (A Wardrop-type principle) Let $f$ be the link flow solution to [TAP-SC], and suppose that

$$
\frac{\partial g_{k}(f)}{\partial f_{a}} \geq 0, \quad \forall a \in \mathcal{A}, \forall k \in \mathcal{K} .
$$

Consider any $O-D$ pair $(p, q)$. Let a route $r \in \mathcal{R}_{p q}$ be defined to be unsaturated if for all $k \in \mathcal{K}$ and all links $a \in \mathcal{A}$ on route $r$,

$$
\frac{\partial g_{k}(f)}{\partial f_{a}}>0 \Longrightarrow g_{k}(f)<0
$$

Assume, without any loss of generality, that in a corresponding route flow solution to [TAP-SC] the first $l$ routes are actually used, and that among these the first $m$ are unsaturated. Then,

$$
c_{p q 1}=\ldots=c_{p q m} \geq c_{p q, m+1} \geq \ldots \geq c_{p q l}
$$

and the unused routes in the $O$-D pair have generalized route costs that are at least as large as that of the used routes in the $O-D$ pair. 
Expressing the route travel costs as

$$
c_{p q r}=\sum_{a \in \mathcal{A}} \delta_{p q r a} t_{a}\left(f_{a}\right), \quad \forall r \in \mathcal{R}_{p q}, \forall(p, q) \in \mathcal{C}
$$

we obtain from (2.8) that

$$
\bar{c}_{p q r}=\sum_{a \in \mathcal{A}} \delta_{\text {pqra }}\left(t_{a}\left(f_{a}\right)+\sum_{k \in \mathcal{K}} \beta_{k} \frac{\partial g_{k}(f)}{\partial f_{a}}\right), \quad \forall r \in \mathcal{R}_{p q}, \forall(p, q) \in \mathcal{C} .
$$

Associated with the generalized route travel costs hence are the generalized link travel costs

$$
\bar{t}_{a}(f) \stackrel{\text { def }}{=} t_{a}\left(f_{a}\right)+\sum_{k \in \mathcal{K}} \beta_{k} \frac{\partial g_{k}(f)}{\partial f_{a}}, \quad \forall a \in \mathcal{A} .
$$

The interpretations of the optimal Lagrange multipliers and the Conditions (2.6)(2.7) depend on the form of the constraint functions $g_{k}$. For example, in the case of simple upper bounds on the link flows $\left(\mathcal{K}=\mathcal{A}\right.$ and $g_{a}(f)=f_{a}-u_{a}, u_{a} \in[0,+\infty]$, for each $a \in \mathcal{A}),(2.18)$ reduces to $\bar{t}_{a}\left(f_{a}\right)=t_{a}\left(f_{a}\right)+\beta_{a}, a \in \mathcal{A}$, and the multipliers $\beta_{a}$ may be associated with the equilibrium queueing delays on the saturated links (i.e., those with flows on their respective upper bounds), and the multipliers $\pi_{p q}$ with the (minimal) sum of total travel cost and queueing delay in each O-D pair; see Miller et al. (1975), Payne and Thompson (1975), Smith (1987), and Larsson and Patriksson (1994). (In the case of capacitated traffic assignment, the constraint functions $g_{a}$ are nondecreasing, and an unsaturated route contains no saturated links.)

The reader should note that the optimal multipliers $\beta$ are not necessarily unique.

\section{SOLVING THE SIDE CONSTRAINED MODEL}

Whenever side constraints are introduced in a traffic assignment model, traditional assignment methods, such as the Frank-Wolfe algorithm and its relatives, either become inapplicable or their efficiency is seriously degraded. In addition, the existing program packages do not possess the ability to take side constraints into account.

However, when considering possible solution principles for the side constrained model, it is most natural to aim at exploiting the efficient solution methods and program packages that are available for the basic model. This immediately leads us to a classical approach for handling complicating constraints: the pricing strategy (e.g., Lasdon, 1970, Ch. 8).

We associate with the side Constraints (2.5) non-negative prices $\beta_{k}, k \in \mathcal{K}$, which define the costs of violating these constraints. Given certain values of these prices, the side constraints are priced-out, i.e., handled implicitly by being included in the objective function. The resulting optimization problem,

$[\operatorname{TAP}(\beta)]$

$$
\min _{f \in F} L(f, \beta)=T(f)+\sum_{k \in \mathcal{K}} \beta_{k} g_{k}(f)
$$

is a standard assignment model (with, in general, a non-separable travel cost function though) and is solvable with most standard methods for the basic model, giving the unique link flow solution $f(\beta)$. (Note that the objective $L(\cdot, \beta)$ is strictly convex with respect to the link flows.) 
The solution to the priced-out problem may be characterized as the solution to a side constrained assignment problem where the right hand sides of the original side constraints are modified through certain perturbations. This result is stated below; it follows immediately from Everett's Theorem (e.g., Lasdon, 1970, Th. 8.3).

Theorem 3. (An Everett-type result for [TAP-SC]) Let $\beta \in \Re_{+}^{|\mathcal{K}|}$ be a price vector. Then the solution $f(\beta)$ to the priced-out problem $[\operatorname{TAP}(\beta)]$ solves the side constrained traffic assignment problem

$[\mathrm{TAP}-\mathrm{SC}(\beta)]$

$\min T(f)$

subject to

$$
\begin{aligned}
\sum_{r \in \mathcal{R}_{p q}} h_{p q r} & =d_{p q}, \quad \\
h_{p q r} & \geq 0, \quad \forall(p, q) \in \mathcal{C}, \\
\sum_{(p, q) \in \mathcal{C}} \sum_{r \in \mathcal{R}_{p q}} \delta_{p q r a} h_{p q r} & =f_{a}, \quad \forall r \in \mathcal{R}_{p q}, \quad \forall(p, q) \in \mathcal{C}, \\
g_{k}(f) & \leq \bar{g}_{k}, \quad \forall k \in \mathcal{K},
\end{aligned}
$$

where

$$
\bar{g}_{k} \stackrel{\text { def }}{=} \begin{cases}g_{k}(f(\beta)), & \text { if } \beta_{k}>0 \\ \max \left\{0, g_{k}(f(\beta))\right\}, & \text { if } \beta_{k}=0\end{cases}
$$

Noting that the priced-out problem $[\operatorname{TAP}(\beta)]$ is equivalent to a standard traffic assignment model with a link travel cost mapping of the form (2.18), a precise relationship between $[\mathrm{TAP}(\beta)]$ and $[\mathrm{TAP}-\mathrm{SC}]$ is obtained when choosing the price vector $\beta$ equal to a vector of optimal Lagrange multipliers for the side Constraints (2.5).

Theorem 4. (An equivalent standard assignment problem) Let $\beta$ be an arbitrary vector of optimal Lagrange multipliers for the side Constraints (2.5). Then the solution set of the standard traffic assignment model with link travel cost mapping

$$
\bar{t}(\cdot)=t(\cdot)+\nabla g(\cdot) \beta
$$

equals that of [TAP-SC].

Proof. The strict convexity of $T$ and the discussion following Theorem 6.5.1 of Bazaraa et al. (1993) yield that $f^{*}=f(\beta)$. The result then follows since the link travel cost mapping of $[\operatorname{TAP}(\beta)]$ is $\nabla L(\cdot, \beta)=t(\cdot)+\nabla g(\cdot) \beta$.

The side constrained assignment model [TAP-SC] may thus be solved as an equivalent, convex, standard traffic equilibrium problem with an appropriately chosen adjustment of the travel costs $t_{a}$; this problem will be referred to as [TAP-A]. Hence, the link travel cost Mapping (3.1) provides a precise statement of the influence of the side constraints on the travel cost perception of the users of the traffic network, and therefore on their route-choice behaviour.

The variational inequality problem corresponding to the first-order optimality conditions of the problem [TAP-A] is to find an $f^{*} \in F$ such that

$$
\bar{t}\left(f^{*}\right)^{\mathrm{T}}\left(f-f^{*}\right) \geq 0, \quad \forall f \in F .
$$


In contrast to the variational inequality formulation [VIP] this problem is symmetric, since its cost mapping is integrable.

The result of Theorem 4 may alternatively be derived from Theorem 3 ; as the price vector tends to a vector of optimal Lagrange multipliers, the solution $f(\beta)$ will, because of the strict convexity of $T$, tend continuously to $f^{*}$, so that the right hand sides $\bar{g}_{k}$ of the side constraints of the problem [TAP-SC $(\beta)$ ] tend continuously to zero and the problem [TAP-SC $(\beta)]$ tends to [TAP-SC]. (Simultaneously, the solution set of the priced-out problem $[\mathrm{TAP}(\beta)]$ tends to that of $[\mathrm{TAP}-\mathrm{SC}]$.

In order to find (near-)optimal values of $\beta$ one may solve the Lagrangean dual problem

[TAP-SCD]

$$
\max _{\beta \geq 0} L(\beta)
$$

where

$$
L(\beta)=\min _{f \in F} L(f, \beta) .
$$

Lagrangean dual problems are typically solved using simple iterative search methods for (essentially) unconstrained optimization. Within a dual solution procedure for [TAP-SCD], the result of Theorem 3 may be utilized for monitoring the progress with respect to the aim of finding a solution to [TAP-SC]. This result also facilitates the finite termination of the dual algorithm when the solution is near-feasible with respect to the side constraints. Clearly, near-feasible solutions are often satisfactory considering the uncertainties in the input data; near-feasibility is also satisfactory when the side constraints are weak, in the sense that they do not need to be fulfilled exactly.

For a link capacity side constrained equilibrium model, Larsson and Patriksson (1994) investigate and evaluate an augmented Lagrangean dualization (i.e., nonlinear pricing) technique for finding optimal values of $\beta$ and establish that it is more efficient than traditional Lagrangean dualization; moreover, for certain instances of augmented Lagrangean schemes, the sequence of dual iterates generated can be shown to converge (at least linearly) although the set of dual solutions is not a singleton in general.

\section{CONCLUSIONS AND FURTHER RESEARCH}

The foundation for the development of models of equilibria based on the inclusion of explicit side constraints is the hypothesis that the additional flow relationships which we want to capture when modelling a real-world traffic problem may be well represented by a set of side constraints. Under this hypothesis, we may establish a close relationship between the improvements of the basic traffic equilibrium model through the introduction of explicit side constraints and generalized travel cost functions, respectively:

(i) In the generalized travel cost approach, the proper cost function to be used has the form (3.1).

(ii) This generalized travel cost function involves gradients of the constraint functions $g_{k}$, which are unknown since they are not formulated explicitly in this approach.

(iii) The proper values of its travel cost parameters $\beta$ are unknown, since the side constrained problem is not solved in this approach. 
Hence, the strategy of using generalized travel costs may be regarded as implicit, which explains, at least partially, why equilibrium models with generalized travel cost functions are difficult to calibrate, and therefore difficult to use in practice.

In contrast, the inclusion of side constraints in the equilibrium model constitutes an explicit approach, in which the physical interpretations of these constraints facilitate the calibration of the model. The strategy of extending the basic model with side constraints also allows a large flexibility in the construction of the model, since the side constraints may be nonlinear as well as non-separable. Thus, the use of side constraints is a direct and general approach for improving the basic model.

To summarize, if explicit side constraints are utilized in a refinement of the basic assignment model, the solution of the resulting model [TAP-SC] automatically produces the travel cost mapping of an equivalent standard traffic equilibrium model. Hence, through a process in which one or more side constrained models are solved, one may derive (i.e., determine the appropriate side constraints) and calibrate (i.e., find the proper coefficients $\beta$ ) adjusted travel cost functions for use in existing transportation analysis tools based on traditional equilibrium models, in order to (indirectly through the cost functions) take into account the additional model components which are described by the side constraints. The solution of an (augmented) Lagrangean dual problem may then be viewed as a means for calibrating these travel cost functions.

The many possibilities for modelling traffic interactions with explicit side constraints, and the strong relationships to equilibrium models with generalized travel costs, motivate the further exploration of this modelling strategy for traffic equilibrium problems. The successful outcome of this exploration relies on cooperations between operations researchers and users ot today's transportation planning systems.

\section{ACKNOWLEDGEMENTS}

The research leading to this report was sponsored in part by the Swedish Transport and Communications Research Board (Grant TFB 92-128-63). The report was written while the second author was on leave at the Department of Mathematics, University

of York, Heslington, York, England. The second author wishes to thank Professor Michael J. Smith of the University of York for his hospitality and many interesting discussions, and the Swedish Transport and Communications Research Board (Grant TFB 93-131-63), the Swedish Institute (Grant 303 GH/MLH), and the Royal Swedish Academy of Sciences for financing the visit.

\section{References}

[1] Bazaraa, M.S., Sherali, H.D., and Shetty, C.M., 1993, "Nonlinear Programming: Theory and Algorithms," second ed., John Wiley \& Sons, New York, NY.

[2] Beckmann, M., McGuire, C.B., and Winsten, C.B., 1956, "Studies in the Economics of Transportation," Yale University Press, New Haven, CT.

[3] Dafermos, S.C., 1972, The traffic assignment problem for multiclass-user transportation networks, Transportation Science 6:73-87. 
[4] Hearn, D.W., 1980, Bounding flows in traffic assignment models, Research Report 80-4, Department of Industrial and Systems Engineering, University of Florida, Gainesville, FL.

[5] Larsson, T., and Patriksson, M., 1994, An augmented Lagrangean dual algorithm for link capacity side constrained traffic assignment problems, in: "Proceedings of the 2nd Meeting of the EURO Working Group on Urban Traffic and Transportation, Paris, France, September 15-17, 1993," vol. 38 of Actes INRETS, F. Boillot, N. Bhouri, and F. Laurent, eds., Institut National de Recherche sur les Transport et leur Sécurité (INRETS), Arcueil, France, pp. 163-199. Also as Report LiTHMAT-R-93-22, Department of Mathematics, Linköping Institute of Technology, Linköping, Sweden, 1993.

[6] Lasdon, L.S., 1970, "Optimization Theory for Large Systems," Macmillan, New York, NY.

[7] Miller, S.D., Payne, H.J., and Thompson, W.A., 1975, An algorithm for traffic assignment on capacity constrained transportation networks with queues. Paper presented at the Johns Hopkins Conference on Information Sciences and Systems, The Johns Hopkins University, Baltimore, MD, April 2-4, 1975.

[8] Nagurney, A., 1993, "Network Economics: A Variational Inequality Approach," Kluwer Academic Publishers, Dordrecht, The Netherlands.

[9] Patriksson, M., 1995, "The Traffic Assignment Problem: Models and Methods," VSP, Utrecht, The Netherlands.

[10] Payne, H.J., and Thompson, W.A., 1975, Traffic assignment on transportation networks with capacity constraints and queueing. Paper presented at the 47th National ORSA Meeting/TIMS 1975 North-American Meeting, Chicago, IL, April 30May 2, 1975.

[11] Sender, J.G., and Netter, M., 1970, Équilibre offre-demande et tarification sur un réseau de transport, Rapport de recherche 3, Département Economie, Institut de Recherche des Transports, Arcueil, France.

[12] Smith, M.J., 1987, Traffic control and traffic assignment in a signal-controlled network with queueing. Paper presented at the Tenth International Symposium on Transportation and Traffic Theory, Boston, MA, 1987.

[13] Wardrop, J.G., 1952, Some theoretical aspects of road traffic research, Proceedings of the Institute of Civil Engineers, Part II, 325-378. 